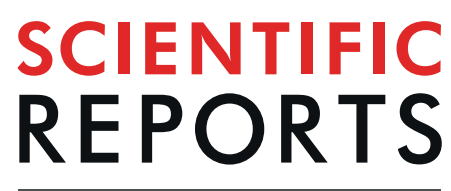

natureresearch

\title{
Gene network approach reveals co-expression patterns in nasal and bronchial epithelium
}

\author{
Kai Imkamp ${ }^{1,2,9 *}$, Victor Bernal $\mathbb{1}^{3,4,9}$, Marco Grzegorzcyk ${ }^{3}$, Peter Horvatovich $\mathbb{1}^{4}$, \\ Cornelis J. Vermeulen $\mathbb{D}^{1,2}$, Irene H. Heijink $k^{1,2,5}$, Victor Guryev ${ }^{2,6}$, Huib A. M. Kerstjens $\mathbb{D}^{1,2}$, \\ Maarten van den Berge ${ }^{1,2,10}$ \& Alen Faiz ${ }^{1,2,5,7,8,10}$
}

Nasal gene expression profiling is a new approach to investigate the airway epithelium as a biomarker to study the activity and treatment responses of obstructive pulmonary diseases. We investigated to what extent gene expression profiling of nasal brushings is similar to that of bronchial brushings. We performed genome wide gene expression profiling on matched nasal and bronchial epithelial brushes from 77 respiratory healthy individuals. To investigate differences and similarities among regulatory modules, network analysis was performed on correlated, differentially expressed and smokingrelated genes using Gaussian Graphical Models. Between nasal and bronchial brushes, 619 genes were correlated and 1692 genes were differentially expressed (false discovery rate $<0.05$, |Fold-change $>2$ ). Network analysis of correlated genes showed pro-inflammatory pathways to be similar between the two locations. Focusing on smoking-related genes, cytochrome-P450 pathway related genes were found to be similar, supporting the concept of a detoxifying response to tobacco exposure throughout the airways. In contrast, cilia-related pathways were decreased in nasal compared to bronchial brushes when focusing on differentially expressed genes. Collectively, while there are substantial differences in gene expression between nasal and bronchial brushes, we also found similarities, especially in the response to the external factors such as smoking.

Chronic obstructive pulmonary disease (COPD) is a chronic inflammatory obstructive disorder of the airways that affects millions of people worldwide ${ }^{1}$. It is a complex and heterogeneous disease caused by many factors, including environmental particles and genetic factors leading to inflammation and metabolic disturbances. Currently COPD is the third most lethal disease worldwide according to World Health Organization ${ }^{1}$. It is mainly caused by inhalation of noxious particles e.g. cigarette smoking, air pollution or indoor cooking, but the disease onset and severity depend on genetic predisposition of the person affected by these environmental circumstances ${ }^{2-4}$.

The initial site of exposure to inhaled substances and particles is the airway epithelium and our group and others have demonstrated that airway gene expression signatures can serve as biomarkers to assess the activity/ severity of COPD and asthma ${ }^{5,6}$. It has also been shown that chronic exposure to tobacco smoke results in both reversible and irreversible changes in bronchial airway epithelial gene expression, a so-called 'airway field of injury ${ }^{7,8}$. Furthermore, we identified a bronchial gene expression signature that is associated with COPD alteration and disease severity with similar gene expression changes in lung tissue affected by $\mathrm{COPD}^{5}$. These data allowed the first finding, which implied that bronchial gene expression obtained by bronchoscopy could be used

${ }^{1}$ University of Groningen, University Medical Center Groningen, Department of Pulmonology, Groningen, The Netherlands. ${ }^{2}$ University of Groningen, University Medical Center Groningen, GRIAC (Groningen Research Institute for Asthma and COPD), Groningen, The Netherlands. ${ }^{3}$ University of Groningen, Bernoulli Institute (JBI), Groningen, The Netherlands. ${ }^{4}$ University of Groningen, Department of Pharmacy, Analytical Biochemistry, Groningen, The Netherlands. ${ }^{5}$ University of Groningen, University Medical Center Groningen, Department of Pathology \& Medical Biology, section Medical Biology, Groningen, The Netherlands. ${ }^{6}$ European Research Institute for the Biology of Ageing, University of Groningen, University Medical Center Groningen, Groningen, The Netherlands. ${ }^{7}$ University of Technology Sydney, Respiratory Bioinformatics and Molecular Biology (RBMB), School of life sciences, Sydney, Australia. ${ }^{8}$ Woolcock Emphysema Centre, Woolcock Institute of Medical Research, University of Sydney, Sydney, NSW, Australia. ${ }^{9}$ These authors contributed equally: Kai Imkamp and Victor Bernal. ${ }^{10}$ These authors jointly supervised this work: Maarten van den Berge and Alen Faiz. *email: k.imkamp@umcg.nl 


\begin{tabular}{|l|l|l|l|}
\hline & All $(\mathbf{N}=77)$ & $\begin{array}{l}\text { Current smokers } \\
(\mathbf{N}=41)\end{array}$ & $\begin{array}{l}\text { Never smokers } \\
(\mathbf{N}=36)\end{array}$ \\
\hline Age, yr & $36.06(16.23)$ & $37.10(15.55)$ & $34.89(17.10)$ \\
\hline BMI kg/m ${ }^{2}$ & $23.73(3.50)$ & $23.91(3.29)$ & $23.52(3.76)$ \\
\hline Gender, Male/Female & $41 / 36$ & $25 / 16$ & $16 / 20$ \\
\hline Pack years**** & $8.68(13.4)$ & $16.30(14.63)$ & 0 \\
\hline FEV $_{1} \%$ predicted & $108.14(10.49)$ & $106.73(10.62)$ & $109.75(10.24)$ \\
\hline Reversibility \% from baseline & $3.82(3.05)$ & $3.82(2.64)$ & $3.82(3.50)$ \\
\hline FEV $_{1} /$ FVC & $83.06(6.37)$ & $81.75(5.97)$ & $84.54(6.57)$ \\
\hline RV \% predicted & $93.74(17.46)$ & $92.83(12.99)$ & $94.78(21.62)$ \\
\hline TLC \% predicted & $104.04(9.42)$ & $102.80(9.56)$ & $105.44(9.19)$ \\
\hline RV/TLC \% predicted & $85.62(12.38)$ & $85.95(8.84)$ & $85.25(15.59)$ \\
\hline
\end{tabular}

Table 1. Clinical characteristics of the NORM study. BMI, body mass index; $\mathrm{FEV}_{1}$, forced expiratory volume in one second; $\mathrm{FEV}_{1} / \mathrm{FVC}$, forced expiratory volume in one second/forced vital capacity; RV, residual volume; TLC, total lung capacity; RV/TLC, residual volume/total lung capacity. The means and standard deviations are shown for continuous variables. ****Independent T-test showed significant difference between the two groups only with a $\mathrm{p}<0.0001$.

\begin{tabular}{|c|c|c|c|}
\hline Gene symbol & rho & p-value ${ }^{* *}$ & FDR $^{* *}$ \\
\hline PSORS1C3 & 0.649 & 0 & 0 \\
\hline TEKT4P2 & 0.658 & 0 & 0 \\
\hline CYP1A1* & 0.652 & 0 & 0 \\
\hline CYP1B1* & 0.774 & 0 & 0 \\
\hline WBSCR27 & 0.653 & 0 & 0 \\
\hline CFD & 0.673 & 0 & 0 \\
\hline SLC44A5 & 0.690 & 0 & 0 \\
\hline NLGN4Y & 0.730 & 0 & 0 \\
\hline SLC7A11* & 0.648 & 0 & 0 \\
\hline TXLNG2P & 0.720 & 0 & 0 \\
\hline TAS2R43 & 0.787 & 0 & 0 \\
\hline GBP3 & 0.880 & 0 & 0 \\
\hline RNU5D-1 & 0.641 & 0 & 0 \\
\hline STEAP1 & 0.727 & 0 & 0 \\
\hline $\mathrm{ABO}$ & 0.745 & 0 & 0 \\
\hline GSTM1 & 0.743 & 0 & 0 \\
\hline GSTT1 & 0.798 & 0 & 0 \\
\hline GUCY1B2 & 0.667 & 0 & 0 \\
\hline HLA-DMB & 0.633 & 0 & 0 \\
\hline HLA-DQA1 & 0.787 & 0 & 0 \\
\hline
\end{tabular}

Table 2. Top 20 genes correlated between nasal and bronchial brushes (FDR $<0.05)$. *Smoking related genes. $* *$ Zero $(0)$ p-values and FDR rate means that the number is smaller than the smallest number that can be represented by a double format in $\mathrm{R}\left(\right.$ i.e. $\left.<10^{-308}\right)$.

to assess the disease activity in COPD. However, bronchoscopy is an invasive procedure with a substantial burden to the patient, preventing its use in large populations as well as frequent sampling.

We previously demonstrated that the nasal epithelium can be used to detect changes in COPD-associated gene expression and showed that the nasal epithelial COPD related gene expression signature partly overlaps with COPD-associated bronchial epithelial gene expression ${ }^{9}$. This strengthens the hypothesis that the upper and lower airways have a common COPD-related gene expression profile, the united 'airway field of injury'. Previously, we and others have shown that nasal and bronchial epithelium have a similar expression profile in smokers and never-smokers. Additionally, we have shown that expression quantitative trait loci (eQTL) are similar between the two compartments ${ }^{10}$. The easy availability to nasal epithelium, nasal gene expression provides the opportunity to assess disease-related phenotypes and determine treatment outcome.

One way to investigate this is the use of gene network modelling. In particular, Gaussian Graphical Models (GGMs), are a widely used network model to study protein ${ }^{11}$, and gene regulatory networks ${ }^{12}$. The GGM consists of nodes (e.g. genes, proteins or metabolites) interconnected by edges if their partial correlation is significantly different from zero. Partial correlations are correlations where the cofounding effects are removed. This is an advantage of GGMs compared to other models such as Relevance Networks ${ }^{13}$ or weighted gene co-expression network analysis (WGCNA $)^{14}$, principal component analysis or clustering, which use the structure obtained from 
A

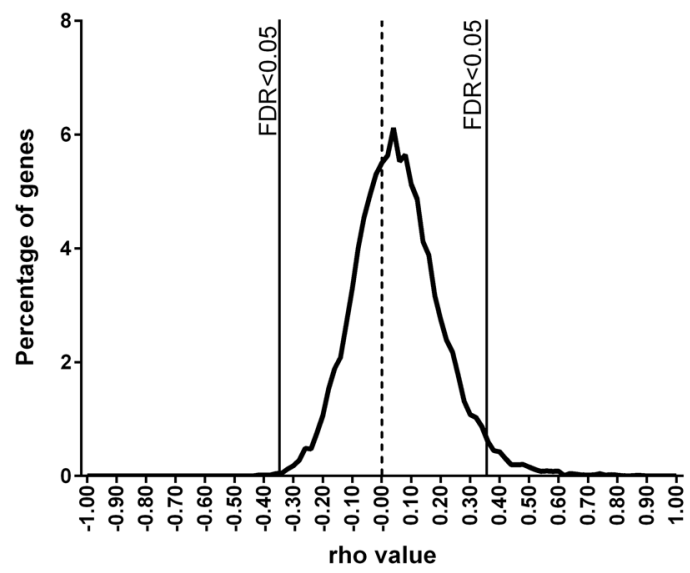

B
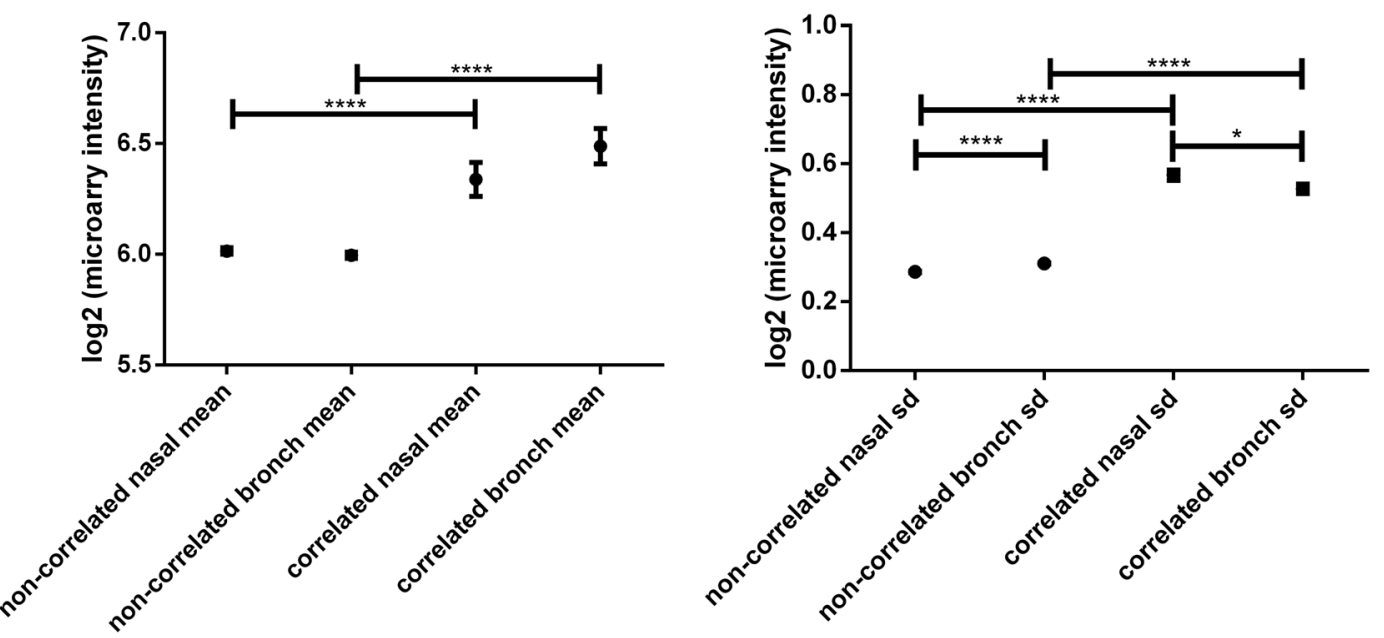

Figure 1. Comparison between nasal and bronchial epithelial brushes. (A) Histogram of Spearman correlation of each concordant gene (FDR-adjusted $\mathrm{p}<0.05$ ) between nasal and bronchial brushes. (B) Comparing mean expression from correlated and non-correlated genes $(\mathbf{C})$ Comparing standard error. $\mathrm{SD}=$ standard error.

Pearson correlation to find similarity patterns because spurious associations are avoided. For example, a common regulator gene might results in similar expression patterns of the regulated genes. These patterns are then indirect and cannot be distinguished from the effect of the regulator. Learning the structure of a GGM (i.e. inferring the edges from data) is computationally feasible even for large networks, and often performs as good as other more demanding network models (e.g. Bayesian Networks) ${ }^{15}$. Some applications in respiratory research include GGMs reconstructed from expression data of asthmatic children ${ }^{16}$, asthma integrated genomic data ${ }^{17}$, COPD phenotypic networks ${ }^{18}$, and asthma gene - single-nucleotide polymorphism (SNP) associations ${ }^{19}$.

In the current study, we aim to investigate to what extent gene expression profiling of nasal brushings are similar to that of bronchial brushings, and to determine whether nasal brushing can be used as a non-invasive biomarker of the lower airways in the study of respiratory diseases. We used GGMs to investigate correlated and differentially expressed genes between the two tissues, and to identify which pathways are similar and which are different.

\section{Results}

Patient characteristics. From the 110 respiratory healthy participants who were enrolled in the study, 77 had matched nasal and bronchial samples. Table 1 shows the clinical characteristics of all participants and the comparison of current and never smokers.

Genes similar between the nose and the bronchus. Spearman correlation analyses, comparing the expression of individual genes between nasal and bronchial brushes, identified 619 genes (3.4\% of total, Benjamini-Hochberg $(\mathrm{BH})^{20}$-adjusted $\left.\mathrm{p}<0.05\right)$ that were significantly correlated between bronchial and nasal samples. Table 2 shows the top 20 genes with correlated expression between the nasal and the bronchial epithelium. As expected, X- and Y-linked genes, smoking-related genes, and genes known to have high genetic contribution to variation in expression, such as $H L A-D R B 1$, were the most significantly correlated genes in this analysis ${ }^{21}$. For the majority of these genes (98\%) expression was positively correlated between nasal and bronchial brushes (Fig. 1A), indicating a subset of genes with strong concordance of their expression between the two 
A

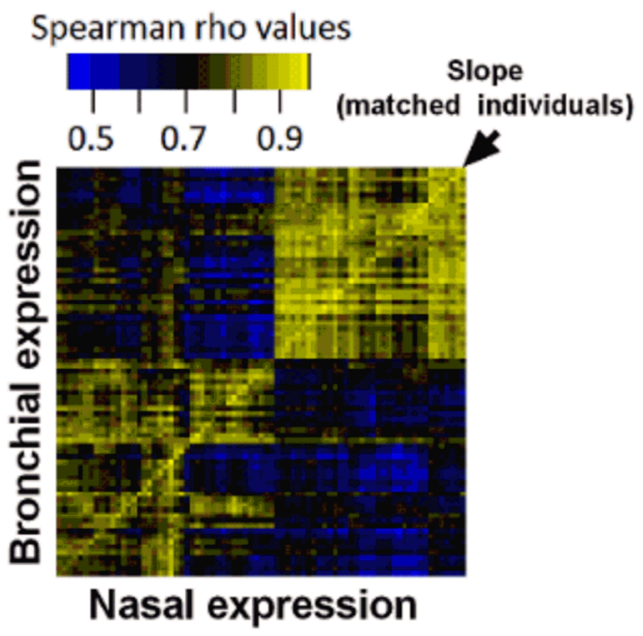

B

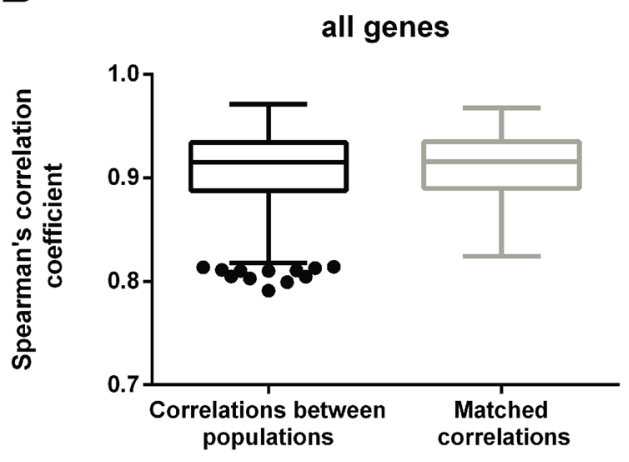

C

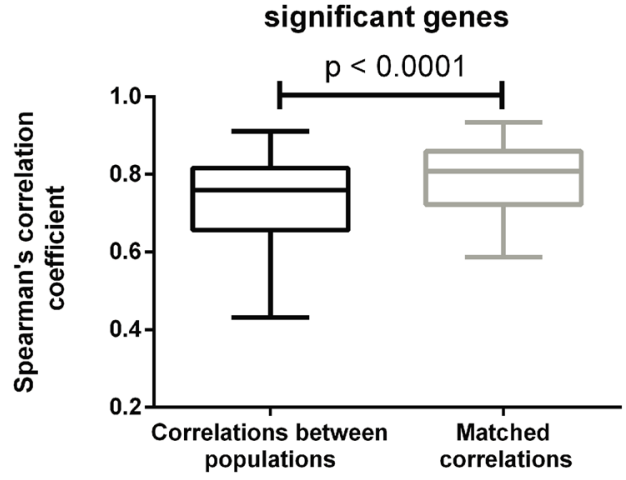

Figure 2. Correlation between nasal and bronchial epithelial brushes. (A) Heatmap showing Spearman correlation of genes between nasal and bronchial brushes (FDR-adjusted $\mathrm{p}<0.05$ ). (B) Correlation between independent and matched samples using all genes and $(\mathbf{C})$ genes which correlate between matched samples of nasal and bronchial brushes.

sampling locations. To confirm that these findings were not by chance, a permutation analysis was conducted $(n=500)$. Indeed, the number of positively correlated genes was always found to be greater with paired samples compared to randomly picked (non-paired) samples $(\mathrm{p}<0.002)$. Furthermore, we found that genes correlated between the two locations have both higher mean expression and greater variation (standard deviation) than non-correlated genes (Fig. 1B,C), indicating that variation of gene expression is required for correlation. We next investigated to what extent the nasal sample reflects an individual's bronchial transcriptional profile rather than a response to environmental insults such as smoking. To this end, we assessed whether the nasal-bronchial relation within a patient was stronger than across patients. We performed this analysis for all genes genome-wide and for the list of 619 correlated genes mentioned above. We found no difference with respect to the nasal-bronchial relation between samples from the nose and bronchus when looking at all genes, while for the 619 correlated genes (CO), the intra-patient nasal bronchial correlations were more correlated than across patients (Fig. 2A-C). This may be explained by the low expression or low variation in expression in the population of the non-correlating genes. These two factors drive the lack of self-correlation as the levels of expression between nasal and bronchial brushes are very similar across all patients.

Network analysis on correlated genes. From these $619 \mathrm{CO}$ genes, we built two GGM networks (one for each tissue) at $\mathrm{BH}$-adjusted $p \leq 0.01$ (Fig. 3A). We found 163 genes $(26.33 \%)$ that are connected in the bronchial network with 156 edges (i.e. significant partial correlations), and 168 (27.14\%) in nasal network with 152 edges. In total 236 genes $(38.12 \%)$ had at least one edge in one of the tissues, from which only 36 genes (15.25\%) have common edges in both compartments.

Figure $3 \mathrm{~B}$ shows an (edge-wise) comparison of the networks via a scatter plot of $\mathrm{BH}$ p-values for the $\mathrm{CO}$ genes. We observed that genes belonging to the same family tend to be interconnected in both tissues. In particular, $H L A-D Q B 1, H L A-D R B 1, H L A-D R B 5$ and $H L A-D Q A 1$ were interconnected to each other. Other gene pairs from the same family are connected as well, namely; (i) SAA1-SAA2, (ii) STEAP1-STEAP2, (iii) RNU5F-1 - RNU5D1, (iv) MT2A - MT1L, (v) CD207-CDH17. Table 3 shows the results of GO enrichment analysis for biological 

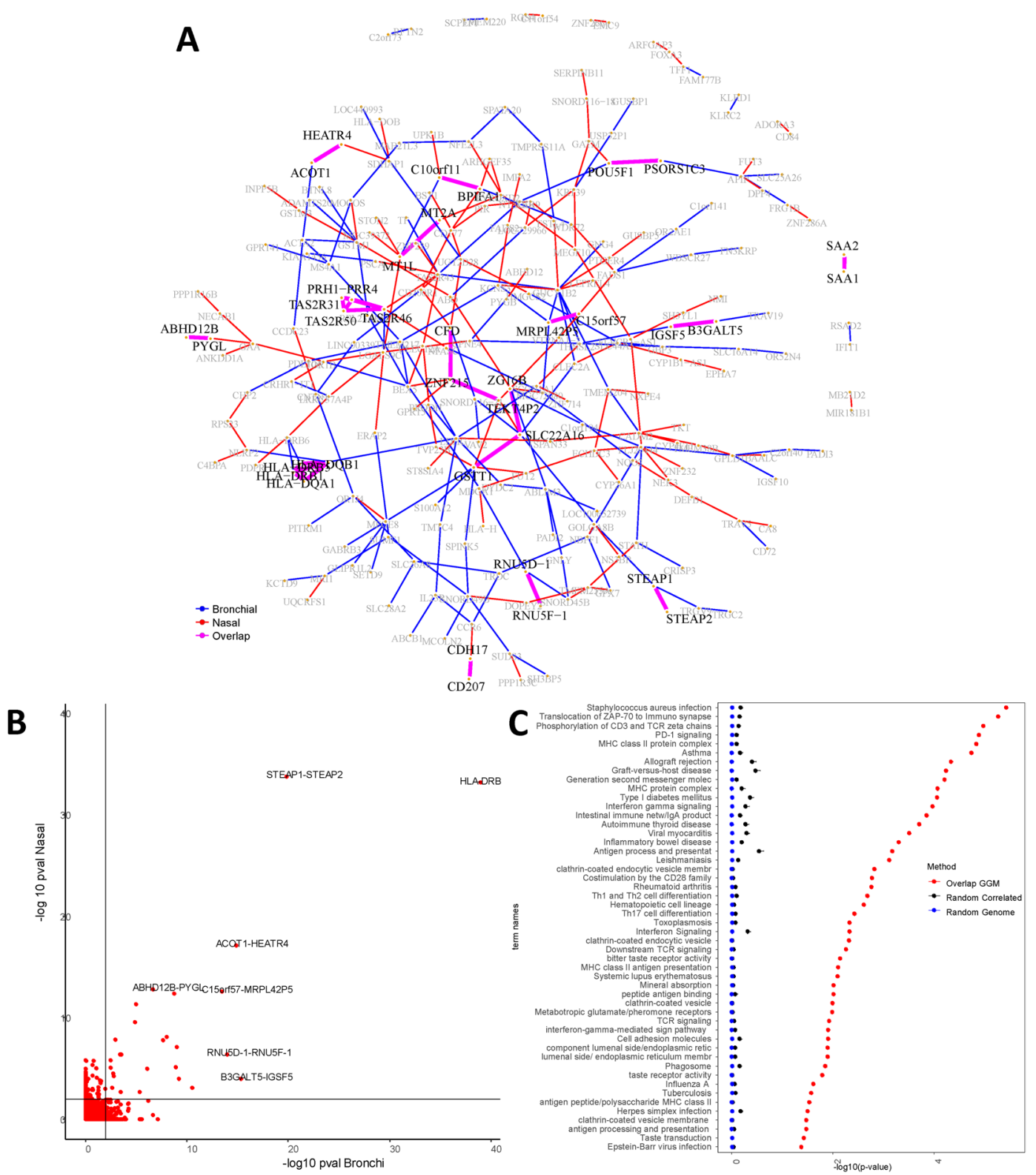

Figure 3. Network analyses for the correlated (CO) genes. (A) GGM network for the CO genes (BH $p \leq 0.01)$. Genes that had no significant connections (partial correlation) were left out of the figure, and the genes connected in only one of the tissues are colored in grey. In blue: edges present in bronchial tissue. In red: edges present in nasal tissue. In magenta: edges present both tissues (Overlapped edges). (B) Scatter plot of the GGM network edges (partial correlation) for the $\mathrm{CO}$ genes. Each red dot represents - $\log _{10}(\mathrm{BH} p)$ of an edge in bronchial tissue (horizontal axis) versus nasal tissue (vertical axis). In light black: the critical value at $\mathrm{BH}$ $p \leq 0.01$. The figure displays the respective gene pairs for the most similar edges. (C) The 50 most significant GOs for the set of Overlapped genes. The enrichment is contrasted against two sets of genes randomly sampled from the CO (619 genes) and from the whole genome (19718 genes). The panel displays the corresponding mean $-\log _{10}$ (p-values) and error bars represent +2 standard errors over the 500 random samples.

processes at False Discovery Rate (FDR) $\leq 0.05$ (Fig. 3C). The enrichment consisted of 56 biological processes mainly related to inflammatory and immunological pathways.

Network analysis on smoking-related genes. Previously, we identified 27 genes that were differentially expressed in current smokers compared to never smokers in both the nasal and bronchial brushes ${ }^{22}$. From these 27 smoking-related genes (SM), we inferred two GGM networks (one for each tissue) at BH-adjusted $\leq 0.05$ (Fig. 4A). We found 8 genes (29.62\%) that are connected in the bronchial network with 4 edges (i.e. having multiple significant partial correlations), and 6 genes (22.22\%) in nasal network with 3 edges. A total of 6 genes (75.00\%, 3 gene pairs) exhibited one common edge between the bronchial and nasal network. Figure 4B shows an (edge-wise) comparison of the networks via a scatter plot of $\mathrm{BH}$ p-values for the SM genes. We observed 


\begin{tabular}{|l|l|l|l|}
\hline Term ID & Term name & p-value & Intersection \\
\hline KEGG:05150 & Staphylococcus aureus infection & $3.63 \cdot 10^{-6}$ & HLA-DQB1, HLA-DRB1, HLA-DQA1, CFD, HLA-DRB5 \\
\hline REAC:202430 & Translocation of ZAP-70 to Immunological synapse & $5.28 \cdot 10^{-6}$ & HLA-DQB1, HLA-DRB1, HLA-DQA1, HLA-DRB5 \\
\hline REAC:202427 & Phosphorylation of CD3 and TCR zeta chains & $1.03 \cdot 10^{-5}$ & HLA-DQB1, HLA-DRB1, HLA-DQA1, HLA-DRB5 \\
\hline REAC:389948 & PD-1 signaling & $1.26 \cdot 10^{-5}$ & HLA-DQB1, HLA-DRB1, HLA-DQA1, HLA-DRB5 \\
\hline GO:0042613 & MHC class II protein complex & $1.43 \cdot 10^{-5}$ & HLA-DQB1, HLA-DRB1, HLA-DQA1, HLA-DRB5 \\
\hline KEGG:05310 & Asthma & $1.77 \cdot 10^{-5}$ & HLA-DQB1, HLA-DRB1, HLA-DQA1, HLA-DRB5 \\
\hline KEGG:05330 & Allograft rejection & $4.48 \cdot 10^{-5}$ & HLA-DQB1, HLA-DRB1, HLA-DQA1, HLA-DRB5 \\
\hline KEGG:05332 & Graft-versus-host disease & $5.63 \cdot 10^{-5}$ & HLA-DQB1, HLA-DRB1, HLA-DQA1, HLA-DRB5 \\
\hline REAC:202433 & Generation of second messenger molecules & $6.11 \cdot 10^{-5}$ & HLA-DQB1, HLA-DRB1, HLA-DQA1, HLA-DRB5 \\
\hline GO:0042611 & MHC protein complex & $8.27 \cdot 10^{-5}$ & HLA-DQB1, HLA-DRB1, HLA-DQA1, HLA-DRB5 \\
\hline
\end{tabular}

Table 3. List of top 10 enriched GOs for the overlapping genes set from the CO genes $(F D R \leq 0.05)$.

that two gene pairs belonging to the same family are connected in both tissues, namely; (i) SAA1-SAA2, (ii) CYP1A1-CYP1B1 and (iii) TFF1- FAM177B. The enrichment was observed for 22 biological processes mainly related to the metabolism of xenobiotics and other $\mathrm{P} 450$ related pathways (Fig. $4 \mathrm{C}$ ). Table 4 shows the results of $\mathrm{GO}$ enrichment analysis for biological processes at $\mathrm{FDR} \leq 0.05$.

Transcription profiles differ between the nose and the bronchus. Next, we investigated which genes drive the differences between the locations. We identified 6,806 expressed in nasal and 6,797 genes expressed in bronchial epithelium $\left(\log _{2}\right.$ (microarray florescence) $<3$ ), with an overlap of $98.2 \%$. Expression of 130 genes was specific to bronchial epithelium, while 145 genes were specifically expressed in nasal epithelium. A differential expression analysis identified 1692 differential expressed genes (DEGs), among which 723 (3.98\%) DEGs with higher and 969 (5.34\%) with lower expression ( $\mid \log$ fold change $\mid>2$, FDR $<0.05$ ) in nasal compared to bronchial brushes. Table 5 shows the 20 most DEGs. Figure 5A shows the DEGs highlighted in red (higher in bronchial brushes) and blue (lower in bronchial brushes) in a scatter plot showing the mean expression in the bronchial and nasal brushes. Figure 5B shows a heatmap of the DEGs.

Network analysis on DEGs. From the 1692 DEGs, we built two GGM networks (one for each tissue) at $\mathrm{BH}$-adjusted $p \leq 0.01$. We found that 821 genes (48.52\%) are connected in the bronchial network with 2642 edges (i.e. significant partial correlations), and 946 (55.91\%) in nasal network with 5291 edges. We found that 1136 genes $(67.13 \%)$ have at least one significant edge in one of the tissues, from which 179 genes $(15.76 \%)$ show common edges, and the remaining 957 genes $(84.24 \%)$ do not exhibit common edges.

Figure 6A shows an (edge-wise) comparison of the networks for each tissue via a scatter plot of BH p-values for the DEGs. This set of 957 genes will be referred to as the genes with non-overlapped edges. The gene ontology (GO) enrichment analysis identified 160 biological processes mainly related to cilium (e.g. cilium part, organization and assembly). The 50 most significant GOs are displayed in Fig. 6B. Table 6 shows the GO enrichment for biological processes at FDR $\leq 0.05$ for the non-overlapped genes.

Cell type decomposition and gene set variation analysis (GSVA) analysis. To investigate the difference in cell-type composition between bronchial and nasal epithelium, we employed markers identified in recent single-cell profiling of human lungs ${ }^{23}$. Interestingly, bronchial epithelium shows higher expression of genes that mark Ciliated and Club cells, while nasal tissue exhibits higher expression of genes characteristic for Goblet cells (Fig. 7A). Within tissue the expression patterns of marker genes is similar between smoker and never smokers where apparent difference can be observed only at the level of individual genes (e.g. MUC5B or CEACAM5). In addition to determine differential pathways expression we have performed GSVA of both tissue type using gene sets attributed to Goblet, and Ciliated cells (Fig. 7B).

\section{Discussion}

In the current study, we used a network-based approach to identify pathways that explain the differences and similarities between the gene expression profiles from nasal and bronchial brushes. This is the first study using GGMs to compare gene networks from the expression data obtained from nasal and bronchial samples. We show that genes involved in inflammatory pathways are retained between the two compartments, while cilia-related genes are lower expressed in the nose. The detoxifying gene expression response of the respiratory tract to cigarette smoking is similar in the nose and bronchus.

When investigating pathways from genes with similar expression in the nose and bronchus, we found genes involved in pro-inflammatory pathways, including the HLA-family, CFD, CD207 and MT2A. The HLA class II molecules have a key function in the adaptive immune response by providing peptides to the antigen receptor of CD4 + T lymphocytes. Several studies have found an association between Human Leukocyte Antigen (HLA) class II genes and asthma, as well as with other allergic diseases, such as allergic rhinitis and atopic dermati$\mathrm{tis}^{22}$. Previous studies have shown similar eosinophilic infiltration (a hallmark of inflammation in asthma) in the nose and bronchus of healthy controls and asthmatics on corticosteroid therapy. Interestingly, although increased inflammation in untreated asthmatics compared to healthy controls was present in both compartments, the inflammation was further enhanced in the bronchus. Furthermore, in vitro studies have shown high 
A

$$
\text { TFFI } \quad \text { FAM }
$$<smiles>C1CCCCC1</smiles><smiles>CNC</smiles>
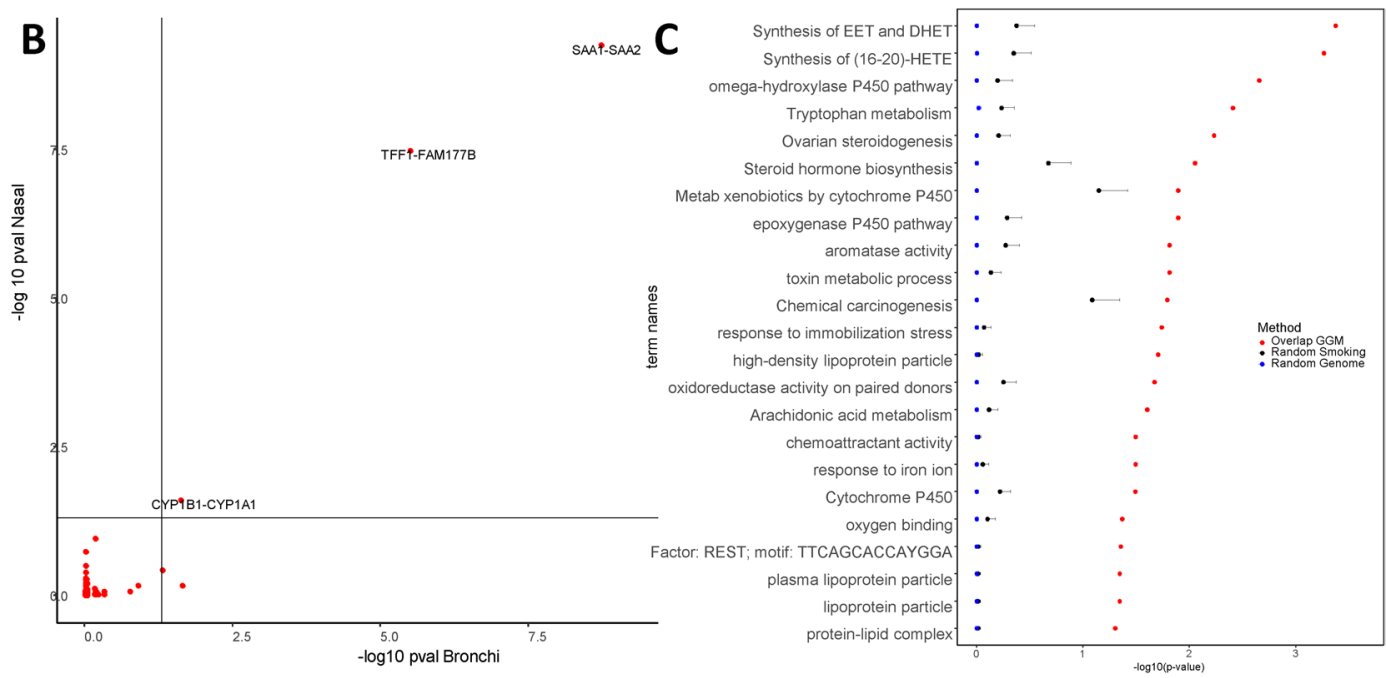

Figure 4. Network analyses for the smoking (SM) genes. (A) GGM network for the SM genes (BH $p \leq 0.05$ ). Genes which had no significant connections (partial correlation) were left out of the figure, and the genes connected in only one of the tissues are colored in grey. In blue: edges present in bronchial tissue. In red: edges present in nasal tissue. In magenta: edges present both tissues (Overlapped edges). (B) Scatter plot of the GGM network edges (partial correlation) for the SM genes. Each red dot represents $-\log _{10}(\mathrm{BH} p)$ of an edge in bronchial tissue (horizontal axis) versus nasal tissue (vertical axis). In light black: the critical value at $\mathrm{BH}$ $p \leq 0.05$. The figure displays the respective gene pairs for the most similar edges. (C) 22 significant GOs for the set of Overlapped genes. The enrichment is contrasted against two sets of genes randomly sampled from the SM (27 genes) and from the whole genome (19718 genes). The panel displays the corresponding mean $-\log _{10}$ (p-values), and error bars represent +2 standard errors over the 500 random samples.

\begin{tabular}{|l|l|l|l|}
\hline Term ID & Term name & p-value & Intersection \\
\hline REAC:2142670 & Synthesis of epoxy (EET) and dihydroxyeicosatrienoic acids (DHET) & 0.000211 & CYP1B1, CYP1A1 \\
\hline REAC:2142816 & Synthesis of (16-20)-hydroxyeicosatetraenoic acids (HETE) & 0.000271 & CYP1B1, CYP1A1 \\
\hline KEGG:00380 & Tryptophan metabolism & 0.000539 & CYP1B1, CYP1A1 \\
\hline KEGG:04913 & Ovarian steroidogenesis & 0.000847 & CYP1B1, CYP1A1 \\
\hline KEGG:00140 & Steroid hormone biosynthesis & 0.00114 & CYP1B1, CYP1A1 \\
\hline KEGG:00980 & Metabolism of xenobiotics by cytochrome P450 & 0.00177 & CYP1B1, CYP1A1 \\
\hline GO:0097267 & omega-hydroxylase P450 pathway & 0.00214 & CYP1B1, CYP1A1 \\
\hline KEGG:05204 & Chemical carcinogenesis & 0.00224 & CYP1B1, CYP1A1 \\
\hline GO:0009404 & toxin metabolic process & 0.00623 & CYP1B1, CYP1A1 \\
\hline GO:0019373 & epoxygenase P450 pathway & 0.0125 & CYP1B1, CYP1A1 \\
\hline
\end{tabular}

Table 4. List of top 10 enriched GOs for the overlapping genes set from the SM genes $(F D R \leq 0.05)$.

concordance in inflammatory responses to the pro-inflammatory stimuli $I L-1 \beta, T N F-\alpha$ and rhinovirus in both nasal and bronchial epithelium ${ }^{24,25}$. The strong concordance in gene expression networks for genes involved in pro-inflammatory pathways (between samples collected in the nose and the bronchus) indicates that the nasal epithelium could be used to study underlying molecular mechanisms of inflammation and to identify easily accessible biomarkers for chronic inflammatory disease classification. 


\begin{tabular}{|l|l|l|l|}
\hline Gene symbol & Log $_{2}$ FC & p-value & FDR \\
\hline PAX6 & 3.270 & $3.09 \cdot 10^{-122}$ & $3.05 \cdot 10^{-118}$ \\
\hline CPA4 & 5.546 & $1.66 \cdot 10^{-122}$ & $3.05 \cdot 10^{-118}$ \\
\hline MUC21 & 4.275 & $1.46 \cdot 10^{-118}$ & $9.62 \cdot 10^{-115}$ \\
\hline GDPD3 & 3.689 & $7.20 \cdot 10^{-113}$ & $3.55 \cdot 10^{-109}$ \\
\hline CERS3 & 3.874 & $3.17 \cdot 10^{-109}$ & $1.25 \cdot 10^{-105}$ \\
\hline XDH & 2.695 & $5.56 \cdot 10^{-109}$ & $1.83 \cdot 10^{-105}$ \\
\hline PAX3 & 2.726 & $2.69 \cdot 10^{-108}$ & $7.59 \cdot 10^{-105}$ \\
\hline VGLL1 & 3.188 & $1.98 \cdot 10^{-107}$ & $4.88 \cdot 10^{-104}$ \\
\hline C8orf34 & -3.702 & $3.55 \cdot 10^{-106}$ & $7.77 \cdot 10^{-103}$ \\
\hline S100A4 & 2.902 & $8.67 \cdot 10^{-105}$ & $1.71 \cdot 10^{-101}$ \\
\hline PI3 & 3.726 & $2.65 \cdot 10^{-104}$ & $4.76 \cdot 10^{-101}$ \\
\hline SIX3 & 2.519 & $1.80 \cdot 10^{-103}$ & $2.96 \cdot 10^{-100}$ \\
\hline SPRR2A & 6.237 & $2.15 \cdot 10^{-103}$ & $3.26 \cdot 10^{-100}$ \\
\hline FGF14 & -4.115 & $2.88 \cdot 10^{-102}$ & $4.05 \cdot 10^{-99}$ \\
\hline CNTN3 & -3.347 & $1.38 \cdot 10^{-101}$ & $1.81 \cdot 10^{-98}$ \\
\hline PAX7 & 2.797 & $8.88 \cdot 10^{-101}$ & $1.09 \cdot 10^{-97}$ \\
\hline GALNT14 & 2.166 & $1.95 \cdot 10^{-100}$ & $2.26 \cdot 10^{-97}$ \\
\hline ANKRD22 & 2.855 & $3.42 \cdot 10^{-100}$ & $3.75 \cdot 10^{-97}$ \\
\hline CD36 & 4.086 & $7.30 \cdot 10^{-100}$ & $7.58 \cdot 10^{-97}$ \\
\hline UCA1 & 5.230 & $9.80 \cdot 10^{-100}$ & $9.66 \cdot 10^{-97}$ \\
\hline
\end{tabular}

Table 5. Top 20 genes differentially expressed between nasal and bronchial brushes $\left(\left|\log _{2} \mathrm{FC}\right|>1.5\right.$, FDR $<0.05$ ).

A

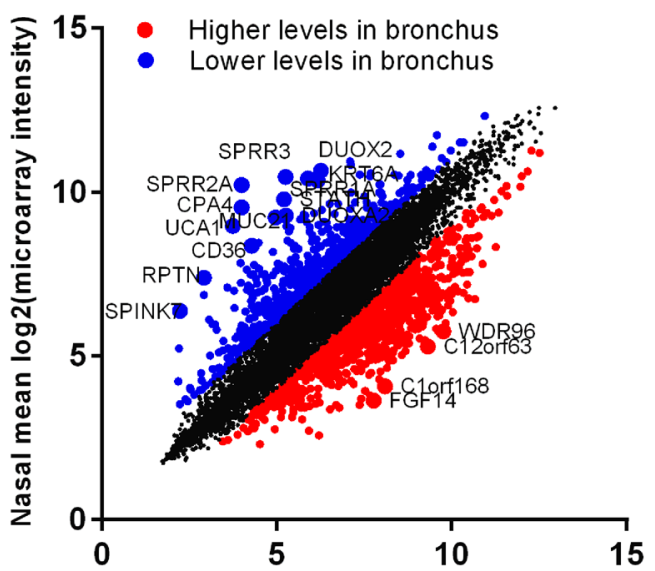

Bronchial mean $\log 2$ (microarray intensity)

\section{B}

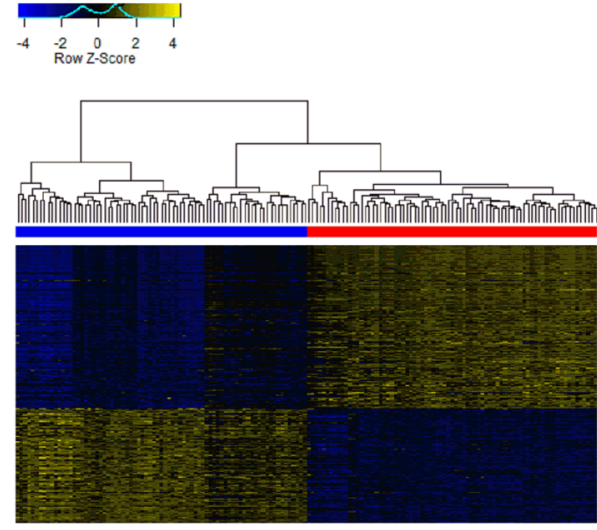

Figure 5. Differences between nasal and bronchial epithelial brushes. (A) Correlation plot of the mean gene expression from nasal and bronchial brushes with genes significantly lower and higher in bronchial brushes highlighted in blue and red, respectively $(\mathrm{FDR}<0.05)$. (B) Heatmap of gene differentially expressed between nasal (blue) and bronchial (red) brushes $\left(\left|\log _{2} \mathrm{FC}\right|>1.5\right.$, FDR $\left.<0.05\right)$.

Furthermore, when looking at pathways concordantly affected by smoking between the nose and the bronchus, genes providing proteins with oxidoreductase activity such as the cytochrome P450 genes (e.g. CYP1A1, $C Y P 1 B 1)$ and $A L D H 1 A 3$, had similar expression between the nose and the bronchus. Previously, it has been shown that genes induced by smoking and having oxidoreductase activity are one of the most rapidly reversible genes upon smoking cessation ${ }^{8}$. Our findings further support the idea of a detoxifying response to tobacco exposure throughout the airways in smokers. Previous studies focusing on ex vivo nasal and bronchial biopsies have shown similar response to cigarette smoke stimulation between the two tissues ${ }^{26}$. Moreover, current and former smokers' bronchial epithelial gene expression has been used to derive and validate a biomarker to detect lung cancer $^{27}$. Subsequently, it was found that lung cancer-associated gene expression was detectable from nasal 
A

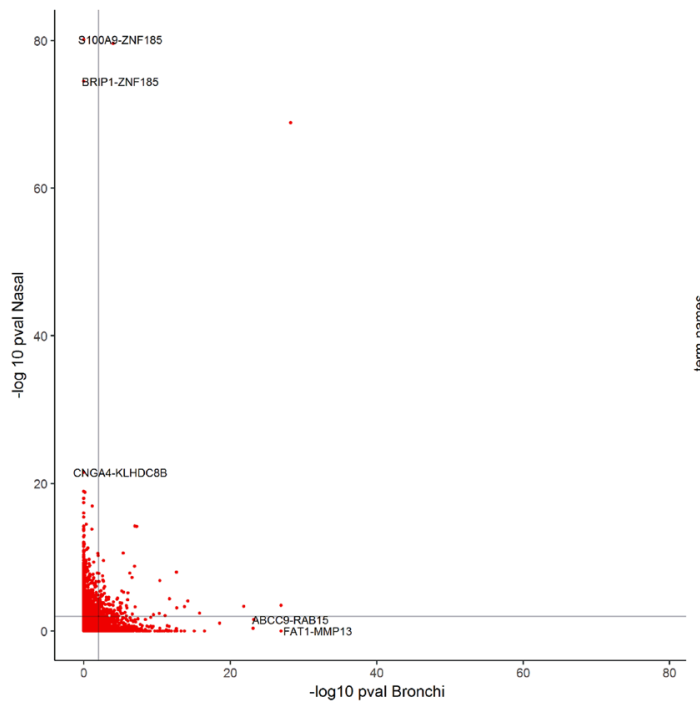

B

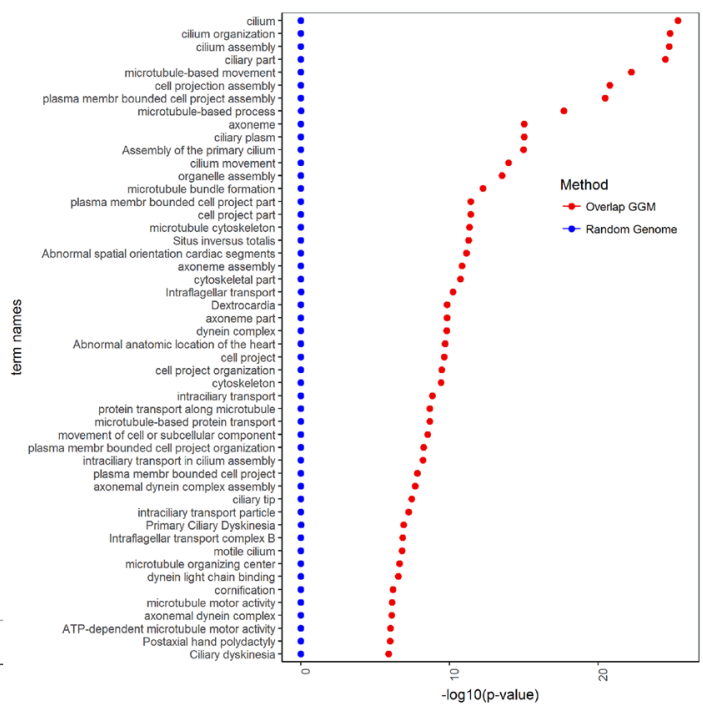

Figure 6. Network analyses for the differentially expressed genes (DEGs). (A) Scatter plot of the GGM network edges (partial correlation) for the DE genes. Each red dot represents $-\log _{10}(\mathrm{BH} p)$ of an edge in bronchial tissue (horizontal axis) versus nasal tissue (vertical axis). In light black: the critical value at $\mathrm{BH} p \leq 0.01$. The figure displays the respective gene pairs for the most similar edges. (B) The 50 most significant GOs for the set of Overlapped genes. The panel displays the corresponding mean $-\log _{10}$ (p-values), and error bars represent +2 standard errors over the 500 random samples.

epithelium, with both tissue showing concordant expression alterations (e.g. regulation of apoptosis and immune system signaling $)^{28}$.

Another gene identified in our analysis is TFF1, which encodes for the trefoil factor family peptides. These peptides are secreted by mucus-producing cells into mucosal surfaces throughout the body and can bind to mucin molecules. It has been shown in mouse studies that TFF1 expression is increased after injury of the airway epithelium, suggesting a role in airway epithelial repair ${ }^{29}$. Another interesting gene is CTNNAL1, a gene that has been associated with cellular growth regulation and may be involved in the recovery of (bronchial) epithelial damage $\mathrm{e}^{30}$.

A number of genes were found to have different expression levels at baseline in nasal and bronchial airway epithelium. However, we still found an overlap between the two networks, indicating that regardless of the different baseline levels of individual genes, there may still be correlations for the gene-sets as a whole between the two compartments. Similar findings have been observed in in vitro models, showing different levels of certain genes between nasal and bronchial epithelial cultures, and nevertheless, these genes still correlated in their response to stimuli $^{25}$. Previous studies focusing on ciliary beat frequency found that this was similar in nasal and bronchial epithelium ${ }^{31}$. However, pathway analysis of differentially expressed genes between nose and bronchus in our study indicated cilia-related pathways to be decreased in nasal compared to bronchial brushes. In line with this observation, it has been shown that the percentage of ciliated cells increases further down the respiratory tract as shown by a previous study ${ }^{32}$. This change in percentage will lead to a shift in cellular composition and an increase of cilia-related gene expression in the lower airways. This finding was confirmed by our GSVA were nasal epithelium showed lower expression for ciliated genes compared to bronchial epithelium. These results support also the high quality of our nasal and bronchial epithelium dataset.

The strength of our study is the use of matched nasal and bronchial samples from a moderately powered dataset. There are also a number of limitations associated with this study. First, from the network analysis perspective GGMs are limited to linear associations (partial correlation). Therefore, if a pair of genes shows a nonlinear relationship, the GGM might not identify these associations. Second, network inference is a multiple testing scenario, i.e. p genes imply performing $p(p-1) / 2$ tests. Here, we have employed BH correction for multiple testing, however, it should be kept in mind that false positives (false edges) might be still present in the network structure regardless of the method depending on the chosen FDR error tolerance. Third, the small number of genes used for the enrichment analysis may have influenced the pathways we found. A subset of the genes with similar gene expression patterns between nasal and bronchial brushing identified in our current analysis are likely due to genetic polymorphisms that have effects on gene expression in general through the whole body not exclusively related to the tissues measured in the current manuscript.

In conclusion, we have shown that although there are differences in expression between the nasal and bronchial brushes, their response to external factors such as smoking seem to be concordant. Therefore, we suggest that the use of nasal brushes as a proxy for the bronchus is suitable to study airway epithelium at baseline and in response to environmental exposures. 


\begin{tabular}{|c|c|c|c|}
\hline Term ID & Term name & p-value & Intersection \\
\hline GO:0005929 & cilium & $4.27 \cdot 10^{-26}$ & $\begin{array}{l}\text { PRKAR2B, DNAH9, MKS1, IFT88, DNAH5, CC2D2A, SPA17, IFT80, SPAG6, MOK, HSPB11, TEKT2, EFHC1, } \\
\text { IFT27, HIF1A, GUCY2F, RPGRIP1L, CCDC114, DNAH11, TTC26, CEP41, B9D1, AKAP3, RSPH4A, MAK, } \\
\text { NME5, IQCG, DNAH1, DNAH6, ALMS1, IFT46, DNAH7, IFT43, DNAL1, BBS9, IFT81, TTC21B, TEKT3, } \\
\text { TAS2R4, TTBK2, TTLL9, TUBG1, MAP1B, CNGA4, DZIP1, TSGA10, TTLL7, DYNC2LI1, CCDC40, SPAG16, } \\
\text { CCDC39, CETN2, WDR78, FAM49B, SPAG17, C8ORF37, WDR19, WDR66, RSPH1, SHANK2, BBS5, DNALI1, } \\
\text { TMEM67, KIF27, AGBL2, TUB, DYNLRB2, TCTN2, ARL13B, TTLL6, DNAI2, CEP19, DNAH12, UNC119B, } \\
\text { DNHD1, GPR157, MAATS1, AGBL4, DYNC2H1, IFT140, CERKL, SNTN, KIF19, TTC30B, DNAH10, CEP290, } \\
\text { EFCAB7, TRAF3IP1, TCTEX1D2 }\end{array}$ \\
\hline GO:0044782 & cilium organization & $1.44 \cdot 10^{-25}$ & $\begin{array}{l}\text { ZMYND10, PRKAR2B, FUZ, MKS1, IFT88, DNAH5, CC2D2A, IFT80, RFX3, PIH1D3, HSPB11, RFX2, TEKT2, } \\
\text { IFT27, RPGRIP1L, CCDC114, TTC26, CEP41, B9D1, C11ORF63, RSPH4A, MAK, NME5, IQCG, DNAH1, } \\
\text { DNAH6, ALMS1, IFT46, DNAH7, CNTRL, IFT43, DNAL1, BBS9, IFT81, TTC21B, TEKT3, TTBK2, FOXJ1, } \\
\text { TUBG1, DZIP1, NEK1, DYNC2LI1, CCDC40, SPAG16, CCDC39, CETN2, SPAG17, WDR19, RSPH1, BBS5, } \\
\text { STK36, TMEM67, KIF27, DNAAF2, PLK1, DNAAF3, DYNLRB2, TCTN2, ARL13B, DNAI2, UNC119B, DNHD1, } \\
\text { CEP97, DYNC2H1, IFT140, KIF19, TTC30B, CEP290, TRAF3IP1, FGFR1OP, TCTEX1D2 }\end{array}$ \\
\hline GO:0060271 & cilium assembly & $1.69 \cdot 10^{-25}$ & $\begin{array}{l}\text { ZMYND10, PRKAR2B, FUZ, MKS1, IFT88, DNAH5, CC2D2A, IFT80, RFX3, PIH1D3, HSPB11, RFX2, TEKT2, } \\
\text { IFT27, RPGRIP1L, CCDC114, TTC26, CEP41, B9D1, C11ORF63, RSPH4A, MAK, NME5, IQCG, DNAH1, } \\
\text { DNAH6, ALMS1, IFT46, DNAH7, CNTRL, IFT43, DNAL1, BBS9, IFT81, TTC21B, TEKT3, TTBK2, FOXJ1, } \\
\text { TUBG1, DZIP1, NEK1, DYNC2LI1, CCDC40, SPAG16, CCDC39, CETN2, SPAG17, WDR19, RSPH1, BBS5, } \\
\text { STK36, TMEM67, KIF27, DNAAF2, PLK1, DNAAF3, DYNLRB2, TCTN2, ARL13B, DNAI2, UNC119B, DNHD1, } \\
\text { CEP97, DYNC2H1, IFT140, TTC30B, CEP290, TRAF3IP1, FGFR1OP, TCTEX1D2 }\end{array}$ \\
\hline GO:0044441 & ciliary part & $2.99 \cdot 10^{-25}$ & $\begin{array}{l}\text { PRKAR2B, DNAH9, MKS1, IFT88, DNAH5, CC2D2A, SPA17, IFT80, SPAG6, MOK, HSPB11, EFHC1, IFT27, } \\
\text { GUCY2F, RPGRIP1L, CCDC114, DNAH11, TTC26, CEP41, B9D1, AKAP3, RSPH4A, MAK, DNAH1, DNAH6, } \\
\text { ALMS1, IFT46, DNAH7, IFT43, DNAL1, BBS9, IFT81, TTC21B, TAS2R4, TTBK2, TUBG1, MAP1B, CNGA4, } \\
\text { DZIP1, DYNC2LI1, CCDC40, SPAG16, CCDC39, CETN2, WDR78, SPAG17, C8ORF37, WDR19, WDR66, RSPH1, } \\
\text { SHANK2, BBS5, DNALI1, TMEM67, AGBL2, DYNLRB2, TCTN2, ARL13B, TTLL6, DNAI2, CEP19, UNC119B, } \\
\text { DNHD1, GPR157, MAATS1, AGBL4, DYNC2H1, IFT140, CERKL, KIF19, TTC30B, DNAH10, CEP290, EFCAB7, } \\
\text { TRAF3IP1, TCTEX1D2 }\end{array}$ \\
\hline GO:0007018 & $\begin{array}{l}\text { microtubule-based } \\
\text { movement }\end{array}$ & $5.74 \cdot 10^{-23}$ & $\begin{array}{l}\text { DNAH9, IFT88, DNAH5, SPA17, IFT80, RFX3, PIH1D3, HSPB11, KIF9, TEKT2, IFT27, HIF1A, CCDC114, } \\
\text { DNAH11, TTC26, RSPH4A, MAK, NME5, KIF20A, DNAH1, DNAH6, IFT46, DNAH7, IFT43, IFT81, TTC21B, } \\
\text { TEKT3, DLGAP5, MAP1B, DYNC2LI1, CCDC40, SPAG16, CCDC39, WDR78, UCHL1, SPAG17, FMN2, WDR19, } \\
\text { AP3S2, WDR66, WDR63, STK36, KIF6, KIF27, DPCD, DYNLRB2, TTLL6, DNAI2, DNAH12, DNHD1, MAATS1, } \\
\text { DYNC2H1, IFT140, KIF19, TTC30B, DNAH10, TRAF3IP1, TCTEX1D2 }\end{array}$ \\
\hline GO:0030031 & cell projection assembly & $1.63 \cdot 10^{-21}$ & $\begin{array}{l}\text { ZMYND10, PRKAR2B, FUZ, MKS1, IFT88, VCL, DNAH5, CC2D2A, IFT80, FSCN1, RFX3, PIH1D3, HSPB11, } \\
\text { RFX2, TEKT2, IFT27, RPGRIP1L, CCDC114, TTC26, CEP41, B9D1, PMP22, C11ORF63, RSPH4A, MAK, NME5, } \\
\text { DPYSL3, IQCG, ARHGEF26, DNAH1, DNAH6, ALMS1, IFT46, DNAH7, CNTRL, IFT43, DNAL1, BBS9, IFT81, } \\
\text { TTC21B, TEKT3, CDC42EP1, TTBK2, FOXJ1, TUBG1, EMP1, DZIP1, TSGA10, NEK1, DYNC2LI1, CCDC40, } \\
\text { SPAG16, CCDC39, CETN2, SPAG17, KIT, WDR19, RSPH1, BBS5, STK36, TMEM67, KIF27, DNAAF2, PLK1, } \\
\text { DNAAF3, DYNLRB2, TCTN2, ARL13B, LPAR3, DNAI2, UNC119B, DNHD1, CEP97, DYNC2H1, IFT140, } \\
\text { TTC30B, CEP290, TRAF3IP1, FGFR1OP, TCTEX1D2, KLHL41 }\end{array}$ \\
\hline GO:0120031 & $\begin{array}{l}\text { plasma membrane bounded } \\
\text { cell projection assembly }\end{array}$ & $3.46 \cdot 10^{-21}$ & $\begin{array}{l}\text { ZMYND10, PRKAR2B, FUZ, MKS1, IFT88, VCL, DNAH5, CC2D2A, IFT80, FSCN1, RFX3, PIH1D3, HSPB11, } \\
\text { RFX2, TEKT2, IFT27, RPGRIP1L, CCDC114, TTC26, CEP41, B9D1, PMP22, C11ORF63, RSPH4A, MAK, NME5, } \\
\text { DPYSL3, IQCG, ARHGEF26, DNAH1, DNAH6, ALMS1, IFT46, DNAH7, CNTRL, IFT43, DNAL1, BBS9, IFT81, } \\
\text { TTC21B, TEKT3, CDC42EP1, TTBK2, FOXI1, TUBG1, EMP1, DZIP1, NEK1, DYNC2LI1, CCDC40, SPAG16, } \\
\text { CCDC39, CETN2, SPAG17, KIT, WDR19, RSPH1, BBS5, STK36, TMEM67, KIF27, DNAAF2, PLK1, DNAAF3, } \\
\text { DYNLRB2, TCTN2, ARL13B, LPAR3, DNAI2, UNC119B, DNHD1, CEP97, DYNC2H1, IFT140, TTC30B, CEP290, } \\
\text { TRAF3IP1, FGFR1OP, TCTEX1D2, KLHL41 }\end{array}$ \\
\hline GO:0007017 & microtubule-based process & $2.01 \cdot 10^{-18}$ & $\begin{array}{l}\text { ZMYND10, DNAH9, IFT88, DNAH5, CC2D2A, SPA17, ASPM, IFT80, RFX3, PIH1D3, HSPB11, KIF9, TEKT2, } \\
\text { IFT27, HIF1A, KATNAL1, CCDC114, DNAH11, TTC26, C11ORF63, RSPH4A, MAK, NME5, KIF20A, IQCG, } \\
\text { DNAH1, DNAH6, CDC20, IFT46, DNAH7, IFT43, DNAL1, IFT81, TTC21B, TEKT3, DLGAP5, GNAI1, TUBG1, } \\
\text { MAP1B, GCC2, DYNC2LI1, CCDC40, SPAG16, CCDC39, PLK2, CETN2, CHEK1, DIXDC1, WDR78, UCHL1, } \\
\text { SPAG17, FMN2, WDR19, AP3S2, WDR66, TPPP3, RSPH1, CCDC78, WDR63, STK36, SPICE1, KIF6, TMEM67, } \\
\text { KIF27, MELK, DNAAF2, DPCD, PLK1, MAP1A, DNAAF3, DYNLRB2, TTLL6, MAP6, DNAI2, CNP, DNAH12, } \\
\text { DNHD1, CEP97, MAATS1, DYNC2H1, IFT140, KIF19, TTC30B, DNAH10, TRAF3IP1, FGFR1OP, TCTEX1D2 }\end{array}$ \\
\hline GO:0097014 & ciliary plasm & $9.55 \cdot 10^{-16}$ & $\begin{array}{l}\text { DNAH9, DNAH5, SPAG6, EFHC1, RPGRIP1L, CCDC114, DNAH11, RSPH4A, DNAH1, DNAH6, DNAH7, } \\
\text { DNAL1, DYNC2LI1, CCDC40, SPAG16, CCDC39, WDR78, SPAG17, WDR66, BBS5, DNALI1, DYNLRB2, } \\
\text { ARL13B, DNAI2, DNHD1, MAATS1, DYNC2H1, IFT140, KIF19, DNAH10, TRAF3IP1, TCTEX1D2 }\end{array}$ \\
\hline GO:0005930 & axoneme & $9.55^{-16}$ & $\begin{array}{l}\text { DNAH9, DNAH5, SPAG6, EFHC1, RPGRIP1L, CCDC114, DNAH11, RSPH4A, DNAH1, DNAH6, DNAH7, } \\
\text { DNAL1, DYNC2LI1, CCDC40, SPAG16, CCDC39, WDR78, SPAG17, WDR66, BBS5, DNALI1, DYNLRB2, } \\
\text { ARL13B, DNAI2, DNHD1, MAATS1, DYNC2H1, IFT140, KIF19, DNAH10, TRAF3IP1, TCTEX1D2 }\end{array}$ \\
\hline
\end{tabular}

Table 6. List of top 10 enriched GOs for the non-overlapping genes from the DEGs $(F D R \leq 0.05)$.

\footnotetext{
Materials and Methods

Study participants and data processing. Data were collected from participants from the study to obtain NORMal values of inflammatory variables from healthy subjects (NORM) NCT00848406 and included healthy smokers and never-smokers. Details on the methods and study design have been previously published ${ }^{22}$. Bronchial and nasal brushings were collected during the same visit, using a Cellebrity bronchial brush (Boston Scientific, Massachusetts, USA) or a Cyto-Pak CytoSoft nasal brush (Medical Packaging Corporation, Camarillo, CA, USA). Samples were then randomized, labeled and run on Affymetrix Human Gene chip ST1.0 arrays as described previously according to manufacturer's instructions (Thermo Fisher Scientific, Waltham, Massachusetts, USA) ${ }^{22}$. Microarray analyses were performed using R (v3.3.2) limma package and normalization was conducted in a single batch using Robust Multi-array Average (RMA). The study procedures were approved by the local medical ethics committee (Medisch Ethische Toetsingsingscommissie or METc) and written informed consent was given by all subjects. All experiments in this study were performed in accordance with relevant Dutch national and international guidelines and regulations.
} 


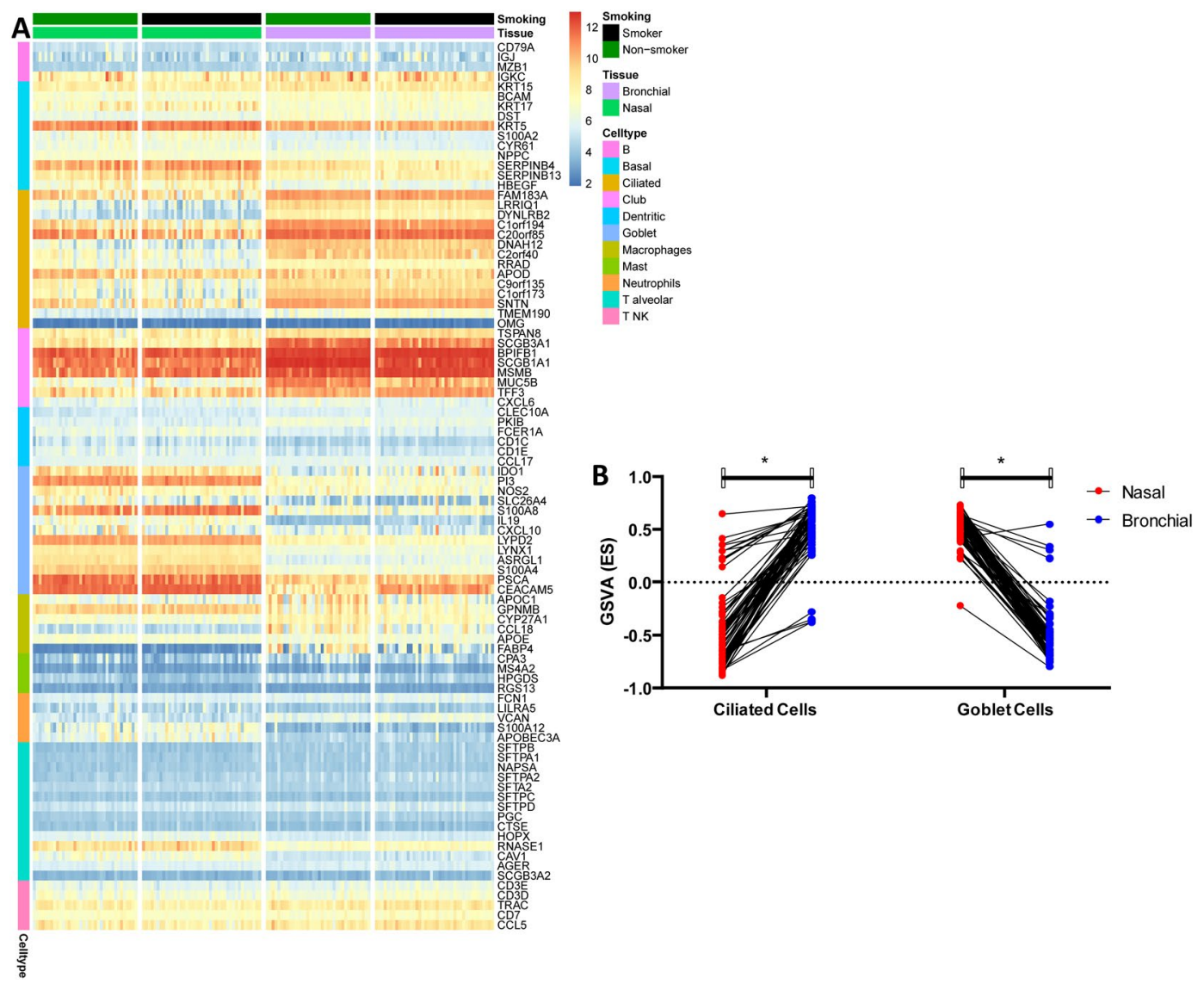

Figure 7. Cell type-specific marker analysis between bronchial and nasal epithelium. In panels of this figure, we investigate the difference in cell-type composition. Panel (A) shows the expression profile of genes identified in recent single-cell profiling of human lungs ${ }^{23}$ in a form of heatmap stratified by tissue type and smoking status. Bronchial epithelium shows higher expression of genes that mark Ciliated and Club cells, while nasal tissue exhibits higher expression of genes characteristic for Goblet cells. Within tissue expression patterns of marker genes is similar between smoker and never smokers where apparent difference can be observed only at the level of individual genes (e.g. MUC5B or CEACAM5). Panel (B) GSVA of ciliated and goblet cell genes from match nasal and bronchial brushes. The scatter plot shows the overall lower GSVA scores of different gene sets attributed to Ciliated cells in nasal samples and the overall higher GSVA scores of gene sets corresponding to Goblet cells.

Gene expression analysis. For the nasal bronchial comparison, a gene was determined to be expressed if the median $\log _{2}$ (normalized microarray florescence intensity) $>3$.

To identify concordant genes where variation among subjects was correlated between sampling locations, we applied a Spearman correlation comparing the expression of individual genes between nasal and bronchial brushes. To assess whether the nasal-bronchial relation within a patient was stronger than across patients, we applied a Spearman correlation on all paired samples and compared this to the average correlation on mismatched pairs. This analysis was conducted on all genes and on the correlated genes.

In order to identify genes differentially expressed between nasal and bronchial brushes, we performed a paired analysis, using a linear model correcting for age, gender and smoking status. Genes were considered significantly differentially expressed if their $\mathrm{BH}$ corrected $\mathrm{p}$-value was $<0.05$ and if they had a $\mid \log$ fold change $\mid>2$.

Network analysis. Three different sets of genes were studied: (i) the set of CO, (ii) twenty seven smoking related genes previously found to be differentially expressed in both the nose and the bronchus ${ }^{22}$ (SM), and (iii) the set of DEGs. The expression data has been $\log _{2}$-tranformed and standardized $(\mathrm{mean}=0, \mathrm{sd}=1)$ such that the data is normal distributed.

As our dataset involves a number of genes that is larger than the sample size, the reconstruction of a GGM (i.e. inferring the partial correlations) from expression data requires a shrinkage approach. This is usually known as a high-dimensional problem. To this end, the network analysis was performed using the R package GeneNet version 1.2.13 ${ }^{12}$ in $\mathrm{R}$ version 3.4.3. The test of significance for each of the GGM's edges (i.e. the partial correlations) was performed with the improved method that we have published recently in Oxford Bioinformatics ${ }^{33}$. This method test the null-hypothesis of no partial correlation between pairs of genes, and has an accurate control of the false 
positives. For networks involving $p$ number of genes there are $p(p-1) / 2$ edges to test which is a multiple testing problem, thus the significance of the edges was adjusted with $\mathrm{BH}$ approach.

Gene ontology enrichment analysis. GO enrichment analysis was performed with the R package gProfileR version $0.6 .4^{34}$, and its default "ontology-focused" multiple testing correction (i.e. g:SCS) ${ }^{35}$. The resulting GO enrichment was assessed by employing a gene sampling procedure. First, we identify which of the GGM's edges were common in both of the analyzed tissues and the corresponding genes were checked for GO enrichment. These pairs of genes (i.e. from the common edges in nasal and bronchial networks) are denoted as the overlapped genes. Second, the GO enrichment from the overlapped genes are compared against the GO enrichment from two others sets of genes (of same size) obtained randomly. The genes of these random sets are sampled from (i) the whole set of protein-coding genes (19718 genes), and from (ii) their corresponding gene sets (e.g. CO, SM or DEG). If the number of connected genes in the network was greater than $50 \%$ its corresponding set (e.g. $50 \%$ of the genes in $\mathrm{CO}, \mathrm{SM}$ or DEG) the sampling was omitted as the two sets are considered similar.

Received: 10 April 2019; Accepted: 13 September 2019;

Published online: 01 November 2019

\section{References}

1. World Health Organization, Global Health Observatory (GHO) data, Top 10 causes of death. Available at, https://www.who.int/gho/ en/.

2. Hobbs, B. D. et al. Genetic loci associated with chronic obstructive pulmonary disease overlap with loci for lung function and pulmonary fibrosis. Nat. Genet. 49, 426-432 (2017).

3. Lamontagne, M. et al. Genetic regulation of gene expression in the lung identifies CST3 and CD22 as potential causal genes for airflow obstruction. Thorax 69, 997-1004 (2014).

4. Artigas, M. S. et al. Sixteen new lung function signals identified through 1000 Genomes Project reference panel imputation. Nat. Commun. 6 (2015).

5. Steiling, K. et al. A dynamic bronchial airway gene expression signature of chronic obstructive pulmonary disease and lung function impairment. Am. J. Respir. Crit. Care Med. 187, 933-42 (2013).

6. Choy, D.F. et al. Gene Expression Patterns of Th2 Inflammation and Intercellular Communication in Asthmatic Airways. Journal of Immunology. 186(3), 1861-1869 (2011).

7. Spira, A. et al. Effects of cigarette smoke on the human airway epithelial cell transcriptome. Proc. Natl. Acad. Sci. USA 101, 10143-8 (2004).

8. Beane, J. et al. Reversible and permanent effects of tobacco smoke exposure on airway epithelial gene expression. Genome Biol. 8 (2007).

9. Boudewijn, I. M. et al. Nasal gene expression differentiates COPD from controls and overlaps bronchial gene expression. Respir. Res. 18, 1-10 (2017)

10. Kontakioti, E., Domvri, K., Papakosta, D. \& Daniilidis, M. HLA and asthma phenotypes/endotypes: A review. Hum. Immunol. 75, 930-939 (2014)

11. Grzegorczyk, M. Extracting protein regulatory networks with graphical models. Proteomics 7(Suppl 1), 51-59 (2007)

12. Schäfer, J. \& Strimmer, K. A Shrinkage Approach to Large-Scale Covariance Matrix Estimation and Implications for Functional Genomics. Stat. Appl. Genet. Mol. Biol. 4, (2005).

13. Butte, A. J. \& Kohane, I. S. Relevance Networks: A First Step Toward Finding Genetic Regulatory Networks Within Microarray Data. In The Analysis of Gene Expression Data (eds Parmigiani, G., Garrett, E. S. \& Irizarry, R. A., Z. S. L.) 428-446 (Springer, New York, NY, 2003), https://doi.org/10.1007/b97411.

14. Zhang, B. \& Horvath, S. A General Framework for Weighted Gene Co-Expression Network Analysis. Stat. Appl. Genet. Mol. Biol., https://doi.org/10.2202/1544-6115.1128 (2005).

15. Werhli, A. V., Grzegorczyk, M. \& Husmeier, D. Comparative evaluation of reverse engineering gene regulatory networks with relevance networks, graphical gaussian models and bayesian networks. Bioinformatics 22, 2523-2531 (2006).

16. Wang, T. et al. FastGGM: An Efficient Algorithm for the Inference of Gaussian Graphical Model in Biological Networks. PLoS Comput. Biol. 12 (2016)

17. Dahlin, A. \& Tantisira, K. G. Integrative systems biology approaches in asthma pharmacogenomics. Pharmacogenomics 13, 1387-1404 (2012).

18. Chu, J. hwa et al. Analyzing networks of phenotypes in complex diseases: Methodology and applications in COPD. BMC Syst. Biol. 8 (2014).

19. Chu, J., Weiss, S. T., Carey, V. J. \& Raby, B. A. A graphical model approach for inferring large-scale networks integrating gene expression and genetic polymorphism. BMC Syst. Biol. 3, 55 (2009).

20. Benjamini, Y. \& Hochberg, Y. Controlling the false discovery rate: a practical and powerful approach to multiple testing. Journal of the Royal Statistical Society 57, 289-300 (1995).

21. Alcina, A. et al. Multiple sclerosis risk variant HLA-DRB1*1501 associates with high expression of DRB1 gene in different human populations. PLoS One 7 (2012)

22. Imkamp, K. et al. Nasal epithelium as a proxy for bronchial epithelium for smoking-induced gene expression and eQTLs. J. Allergy Clin. Immunol, https://doi.org/10.1016/j.jaci.2018.01.047 (2018).

23. Vieira Braga, F. A. et al. A cellular census of human lungs identifies novel cell states in health and in asthma. Nat. Med. 25 (2019).

24. Chanez, P. et al. Comparison between nasal and bronchial inflammation in asthmatic and control subjects. Am. J. Respir. Crit. Care Med. 159, 588-595 (1999).

25. McDougall, C. M. et al. Nasal epithelial cells as surrogates for bronchial epithelial cells in airway inflammation studies. Am. J. Respir. Cell Mol. Biol. 39, 560-568 (2008).

26. Talikka, M. et al. The Response of Human Nasal and Bronchial Organotypic Tissue Cultures to Repeated Whole Cigarette Smoke Exposure. Int. J. Toxicol. 33, 506-517 (2014).

27. Whitney, D. H. et al. Derivation of a bronchial genomic classifier for lung cancer in a prospective study of patients undergoing diagnostic bronchoscopy. BMC Med. Genomics, https://doi.org/10.1186/s12920-015-0091-3 (2015).

28. AEGIS Study Team. Shared Gene Expression Alterations in Nasal and Bronchial Epithelium for Lung Cancer Detection. J. Natl. Cancer Inst. 109 (2017).

29. Greeley, M. A., Van Winkle, L. S., Edwards, P. C. \& Plopper, C. G. Airway trefoil factor expression during naphthalene injury and repair. Toxicol. Sci. 113, 453-467 (2009).

30. Xiang, Y. et al. Identification of Transcription Factors Regulating CTNNAL1 Expression in Human Bronchial Epithelial Cells. PLoS One 7, e31158 (2012). 
31. Low, P. M., Luk, C. K., Dulfano, M. J. \& Finch, P. J. Ciliary beat frequency of human respiratory tract by different sampling techniques. Am Rev Respir Dis 130, 497-498 (1984).

32. Raman, T. et al. Quality control in microarray assessment of gene expression in human airway epithelium. BMC Genomics 10, 493 (2009).

33. Bernal, V., Bischoff, R., Guryev, V., Grzegorczyk, M. \& Horvatovich, P. Exact hypothesis testing for shrinkage-based Gaussian graphical models. Bioinformatics 1-7, https://doi.org/10.1093/bioinformatics/btz135 (2019).

34. Reimand, J. et al. g:Profiler-a web server for functional interpretation of gene lists (2016 update). Nucleic Acids Res. 44, W83-W89 (2016).

35. Reimand, J., Kull, M., Peterson, H., Hansen, J. \& Vilo, J. G:Profiler-a web-based toolset for functional profiling of gene lists from large-scale experiments. Nucleic Acids Res. 35, 193-200 (2007).

\section{Acknowledgements}

This work was supported by the Data Science and System Complexity Centre (DSSC) of the University of Groningen.

\section{Author contributions}

A.F., M.v.d.B. conceived and supervised the project. K.I., M.v.d.B., HamK performed the cell sampling from patients. K.I., I.h.H. and M.v.d.B. collected clinical metadata. K.I. and C.j.V. performed the microarray measurement. V.G. performed microarray data pre-processing and analysis. V.B., M.G. and P.H. performed the G.G.M. network and G.O. analyses. K.I., V.B., V.G. and A.F. prepared the figures. K.I., V.B., M.G., C.j.V., P.H., I.h.H., M.v.d.B. and A.F. wrote the manuscript with input from all authors.

\section{Competing interests}

The authors declare no competing interests.

\section{Additional information}

Correspondence and requests for materials should be addressed to K.I.

Reprints and permissions information is available at www.nature.com/reprints.

Publisher's note Springer Nature remains neutral with regard to jurisdictional claims in published maps and institutional affiliations.

(c) (i) Open Access This article is licensed under a Creative Commons Attribution 4.0 International License, which permits use, sharing, adaptation, distribution and reproduction in any medium or format, as long as you give appropriate credit to the original author(s) and the source, provide a link to the Creative Commons license, and indicate if changes were made. The images or other third party material in this article are included in the article's Creative Commons license, unless indicated otherwise in a credit line to the material. If material is not included in the article's Creative Commons license and your intended use is not permitted by statutory regulation or exceeds the permitted use, you will need to obtain permission directly from the copyright holder. To view a copy of this license, visit http://creativecommons.org/licenses/by/4.0/.

(C) The Author(s) 2019 